\title{
A new template for MRI-based intracavitary/ interstitial gynecologic brachytherapy: design and clinical implementation
}

\author{
Silvia Rodriguez Villalba, MD, PhD', Jose Richart Sancho, MD, PhD', Antonio Otal Palacin, MD, PhD', \\ Jose Perez Calatayud, MD, PhDl,2, Manuel Santos Ortega, MD, PhDl \\ 'Radiotherapy Department, Hospital Clínica Benidorm, Benidorm, Alicante, ${ }^{2}$ Radiotherapy Department, La Fe University and Polytechnic \\ Hospital, Valencia, Spain
}

\begin{abstract}
Purpose: To describe the potential clinical use of a new brachytherapy applicator for gynecological tumors, with special attention to locally advanced cervical carcinoma. This device allows the combination of intracavitary radiotherapy and MRI-compatible transperineal interstitial needles. The design of this template addresses the disadvantages of currently commercially available templates: the inability of the intracavitary component to reach deep into the cervix (MUPIT), and the MRI-incompatibility of these templates (MUPIT and Syed), which necessitates use of CT imaging for the dosimetry.

Material and methods: The newly developed Benidorm Template applicator allows titanium needles in a template with straight and angled holes to provide different angles of divergence to be used with currently existing MRI-compatible intrauterine tubes. It can provide total coverage of the craniocaudal and lateral extension of the tumor (intrautherus, parametrial, and paravaginal). This method is mainly indicated in advanced cervical carcinoma with bulky parametrial invasion (medial or distal), with bulky primary disease that responds poorly to external beam radiotherapy extensive paravaginal involvement (tumor thickness greater than $0.5 \mathrm{~cm}$ ) extending to the middle or lower third of the vagina, or for disease that has invaded the bladder or rectum (stage IVA).

Results: Between April 2013 until December 2014, we treated 15 patients with locally advanced cervical carcinoma employing the Benidorm Template. The median dose at $\mathrm{D}_{90}$ for the CTV was $79.8 \mathrm{~Gy}(71.5-89.9 \mathrm{~Gy})$, at $\mathrm{D}_{2 \mathrm{cc}}$ for the bladder it was 77.6 Gy (69.8-90.8 Gy), and at $\mathrm{D}_{2 \mathrm{cc}}$ for the rectum it was 71.9 Gy (58.3-83.7 Gy). Values expressed in EQD2, assuming $\alpha / \beta$ of 10 for CTV and 3 for OAR.

Conclusions: This new applicator allows the use of MRI-based dosimetry, thus providing the advantages of MRI volume definition. As such, it facilitates determination of complete intracavitary and interstitial CTV coverage and the sparing of normal tissues.
\end{abstract}

Key words: brachytherapy, cervical carcinoma, intracavitary, interstitial, MRI based dosimetry.

\section{Purpose}

The use of the modern MRI imaging techniques has changed the prospects for the treatment of cervical carcinoma and has become a valuable tool in the brachytherapy (BT) component [1-3]. The American Brachytherapy Society (ABS) and the Groupe Européen de Curiethérapie and the European Society for Radiotherapy and Oncology (GEC-ESTRO) have recommended MRI as the preferred image modality for image guided brachytherapy (IGBT) [4-6]. In locally advanced cervical carcinoma with moderate extension to the parametrium, combined endocavitary and interstitial applicators (Vienna or Utrecht applicators, Nucletron, an Elekta company, Elekta AB, Stockholm, Sweden $[7,8])$ are appropriate. However, these have coverage limitations in patients with more advanced disease or in those who respond poorly to external beam radiotherapy (EBRT). In such cases, interstitial templates such as the MUPIT (Martinez Universal Perineal Interstitial Template, Nucletron, an Elekta company, Elekta AB, Stockholm, Sweden) or the Syed Template (Best Medical International, Inc. Springfield, VA, USA) have been recommended $[9,10]$.

The current commercially available interstitial templates have inherent disadvantages: a) some do not allow addition of an intracavitary component, and b) they are 
incompatible with MRI and therefore require use of CT dosimetry. In an attempt to combine the technical advantages of MUPIT and Utrecht applicator and MRI-based planning, we have developed a new intracavitary/interstitial applicator compatible with MRI.

In contrast to the existing multi-interstitial templates (MUPIT and Syed), the Benidorm Template is completely compatible with MRI, thus avoiding the artifacts and image distortion produced by stainless components and improving the MRI image for treatment volume design and dosimetry. Use of this device facilitates adherence to the recommendations of ABS/GEC ESTRO for IGBT. The purpose of this work is to present the design of this new applicator and to describe clinical indications, the clinical procedure, and the advantages of MRI dosimetry.

\section{Material and methods}

\section{Applicator description}

The Benidorm Template (Lorca Marín S.A, Murcia, Spain) uses currently existing Nucletron-Elekta MRI-compatible intrauterine tubes; these 15,30 , and 45 grade tubes are 4,6 , or $8 \mathrm{~cm}$ long and allow delivery of a large central dose. This device can also use 20 or $24 \mathrm{~cm}$ long titanium needles to cover the disease in all directions. The intracavitary component (IC) helps to straighten the uterus and to maintain the geometry of the needles.

The Benidorm Template has 12 rows $1.1 \mathrm{~cm}$ apart (measured from center to center of each needle) to introduce straight and angled titanium needles. It has 7 rows, in which there are 35 straight holes for parallel needle placement and 5 rows, in which there are 16 angled holes $\left(7^{\circ}\right)$ (Figure 1A). This template allows coverage of the distal parametrium (up to $4.5 \mathrm{~cm}$ from the middle of the IC tube) and the entire vagina (Figure 1B). It has also the possibility to include a vaginal cylinder $2.4 \mathrm{~cm}$ in diameter; these cylinders are available in lengths from $4-12 \mathrm{~cm}$ to accommodate different vaginal lengths, with 8 positions of needles in its surface (Figure 1C and D). Plastic obturator tracks fasten the needles avoid displacement (Figure 1E). A second plate of the exactly same design covers the first in order to achieve adequate docking and to prevent displacement of the components of the applicator (Figure 1F). This second plate also can hold a central needle when the introduction of the intrauterine tube is not possible (Figure $1 \mathrm{G}$ and $\mathrm{H}$ ).

This new template takes the well-known advantages of the MUPIT [11] and complements them with an intrauterine tube, additional needle holes, and compatibility with MRI for CTV-OAR delineation.

\section{Clinical indications}

This novel template is specially indicated in locally advanced cervical carcinomas with bulky parametrial extension (medial or distal), bulky primary disease or disease with poor response to EBRT, extensive paravaginal involvement (tumor thickness greater than $0.5 \mathrm{~cm}$ ) extending to middle and lower third of the vagina, or disease that has invaded the bladder or rectum (stage IVA).

In addition, this applicator can be used in patients who are not candidates for an intrauterine component because of unfavorable topography after EBRT and chemotherapy, narrow vaginas, primary tumors of the vagina, or post-hysterectomy recurrences or recurrences in a previously irradiated area.

\section{Clinical procedure}

A pre-implant MRI with the vaginal obturator is done one week before the BT procedure. T2 weighted MRI images in the axial, coronal, and sagittal planes are taken (Figure 2). Using these images, the number, position, and depths of the needles are defined, always taking into account that an offset of $1 \mathrm{~cm}$ from the tip is given to define the first dwell position. This means that the depth is considered appropriate if the CTV is covered in its entirety with up to a $1 \mathrm{~cm}$ superior margin. Implantation of the applicator is done under general or spinal anesthesia. The patient is placed in the dorsal lithotomy position. A Foley tri-lumen catheter is placed in the bladder and inflated with $7 \mathrm{cc}$ of saline solution. An intrauterine tube is brought into the uterine cavity. The obturator (vaginal cylinder) of the template assembly is placed in the vagina, so that it fits against the cervix and maintains that position.

Different lengths of vaginal cylinders are available to match different anatomies. The plate is positioned against the perineum, and the template is fixed to the perineal skin by four or six stitches. With the obturator in the vagina and the template fixed to the perineum, titanium needles (in our case $20 \mathrm{~cm}$ long) are inserted through the holes of the template and through the perineum into the desired target; the obturator tracks exactly the depth previously defined with the pre-implant MRI. If the lateral dimension must be increased, the oblique holes are used. After the implant, a second plate is placed covering the one fixed to the perineum to prevent longitudinal displacement of the needles.

In the MRI (General Electric 1,5 T, Milwaukee, WI, USA) we use a T2 sequence. To visualize the applicator and titanium needles, a T1 3D radio-frequency Spoiled Gradient recalled Echo (SPGR) [12] sequence is also used (Figure 3). Normal saline solution (50 cc) is injected into the bladder to help in volume definition.

Due to the dose characteristics involved in using a single interstitial implant and a single prescription dose over the CTV, we delineate the OARs (rectum and bladder) and the CTV. The CTV is defined taking into account bright regions of tumor seen in T2 sequences, initial image MRI studies, clinical examination at the first consultation, and by examination under anesthesia at the time of the implant $[13,14]$.

Intrauterine tandem and needle reconstruction has been object of another work dedicated to the medical physics aspects of the Benidorm Template. Briefly, the reconstruction was determined by the relationship of the free length of the needles to MRI markers specific to the Benidorm Template [15]. For the intrauterine tandem, the reconstruction was done using MRI dummies described previously [16]. Dosimetry with this template is calculated in the same manner as with MUPIT-based CT planning. The advantage in using MRI instead of CT is the more adequate CTV definition that typically results in 

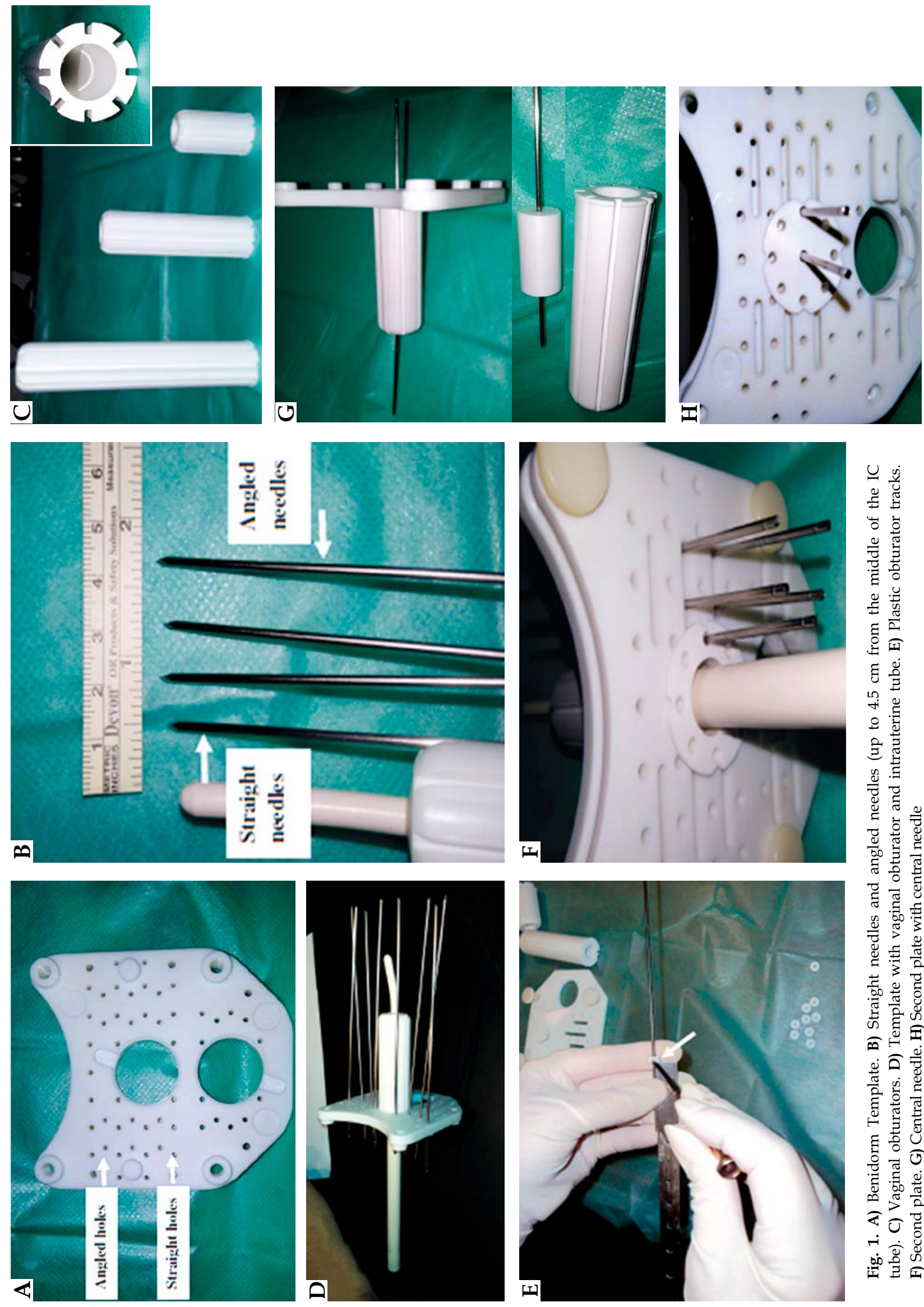

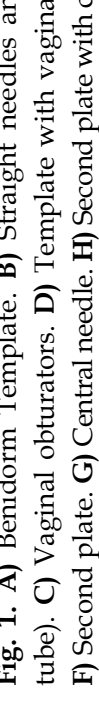



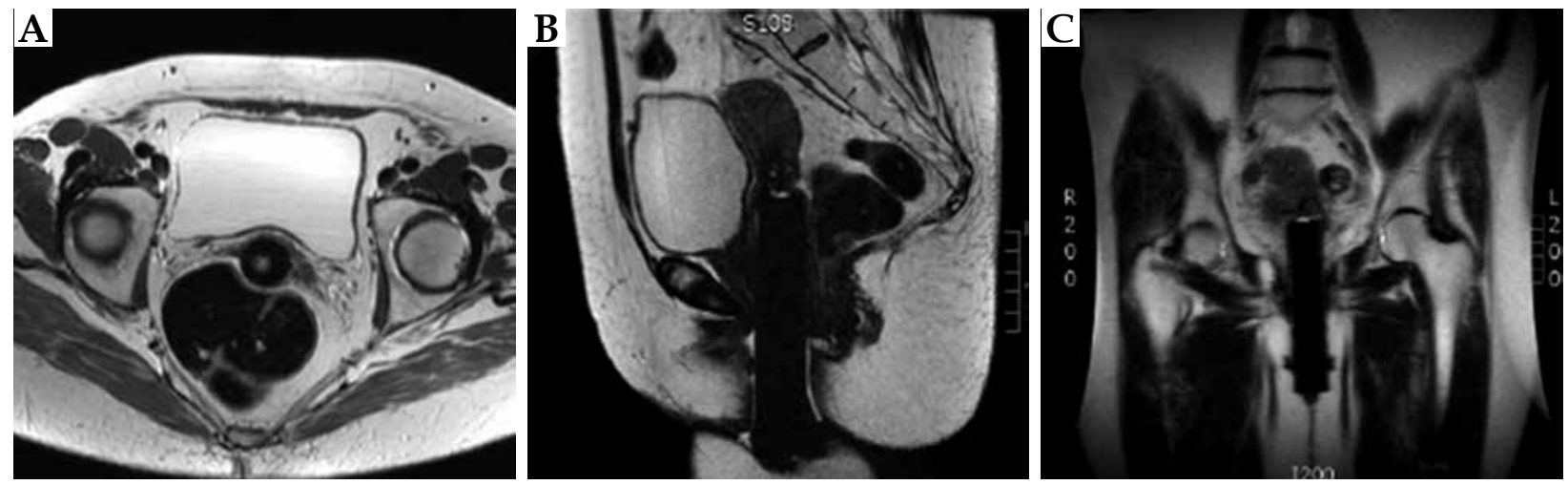

Fig. 2. Pre implant MRI with vaginal obturator. A) Axial plane. B) Sagittal plane. C) Coronal plane
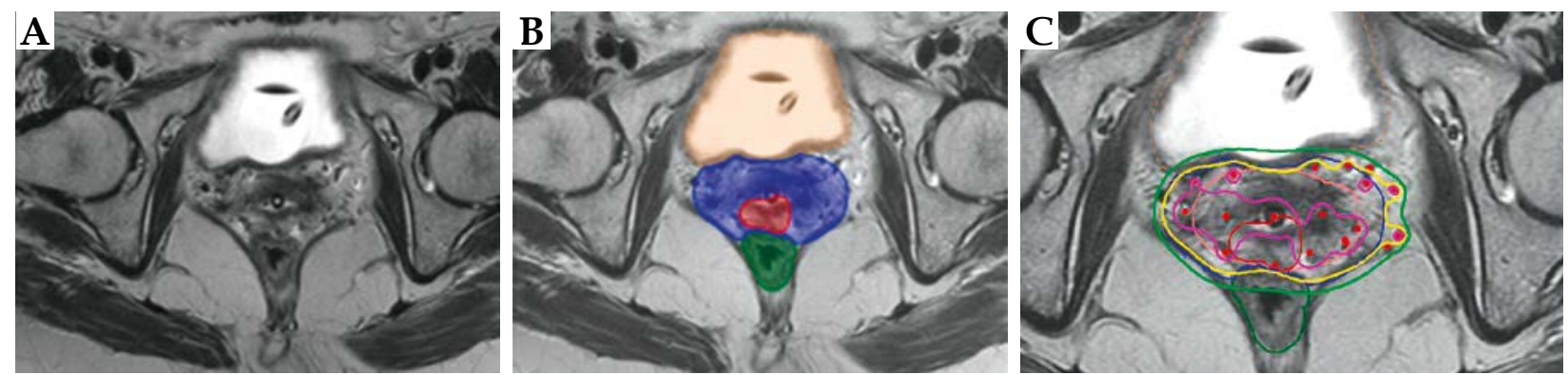

Fig. 3. Planning MRI. T2 sequence. A) Axial plane. B) GTV (red), CTV (blue) and OAR. C) Isodoses: 150\% (magenta), 100\% (yellow), $80 \%$ (green)

a lower CTV volume than that derived using CT. Activation was done inside the volume of CTV. The normalization and prescription were based on the average of the surface CTV points. The optimization was done first geometrically to minimize overdose volumes, and then the result was modified manually to maximize bladder and rectum sparing. Independent calculation verification was performed importing the points, dwell positions, and dwell times from the treatment planning system to a home-made spreadsheet [17].

For these patients, the radiation oncology treatment strategy included an EBRT component that is delivered before BT to reduce tumor volume (46-50.4 Gy to the pelvis with conventional fractionation). We tried to limit the overall treatment time to less than 8 weeks. The customary BT approach adopted by our department was used: six 4 Gy fractions prescribed to CTV and given in four days, six hours apart. The bladder was filled with $50 \mathrm{cc}$ of saline solution before each fraction to reproduce the planned treatment defined in the MRI. The implantation and first treatment were completed on day one. Total doses were normalized to 2 Gy per fraction equivalent doses (EQD2), assuming $\alpha / \beta$ of 10 for CTV and 3 for OAR as recommended $[5,6]$.

This fraction size was chosen to decrease the risk of late toxicity that might result from the biologic effect of a larger fractional dose and the fact that two fractions were administered daily $[18,19]$. We performed a single application implantation with multifractionated HDR irradiation. The planning aim was to deliver at least 75-85 Gy EQD2 $(\alpha / \beta=10)$ to de CTV $D_{90}$. Dose constraints applied to the OARs were based on the EMBRACE protocol for cervix, using a max- imum dose to $\mathrm{D}_{2 \mathrm{cc}}$ rectum of 70-75 Gy EQD2 $(\alpha / \beta=3)$ and 90 Gy EQD2 ${ }_{(\alpha / \beta=3)}$ to the $D_{2 c c}$ of the bladder [20].

Implant needles were removed without anesthesia immediately after the treatment was completed. Routine antibiotics, continuous intravenous analgesia, and antiembolism prophylaxis were prescribed during the treatment period. The patients were provided with antibiotics and analgesics, and discharged the same day that the implant was removed.

\section{Results}

The objective of this manuscript is to describe the CTV coverage achieved with the IC/interstitial Benidorm Template applicator and to demonstrate the improvement in Isodoses: 150\% (Magent), 100\% (yellow), 90\% (green) volume definition that was possible through the use of MRI. In a future phase of this project, the clinical follow-up and tolerance results will be analyzed and compared with our previous results with CT-based dosimetry using the MUPIT applicator.

Between April 2013 until December 2014, we used the Benidorm Template to place 15 implants in patients with locally advanced cervical carcinoma (8 stage IIB, 3 stage III, and 4 stage IVA). Tumor characteristics, CTV $\mathrm{D}_{90}$, and bladder and rectum $\mathrm{D}_{2 c c}$ of these patients are listed in Table 1. Median $\mathrm{D}_{90}$ for CTV was 79.8 Gy (71.5-89.9 Gy EQD2 $(\alpha / \beta=10))$; median $\mathrm{D}_{2 \mathrm{cc}}$ for bladder was $77.6 \mathrm{~Gy}$ (69.8$\left.90.8 \mathrm{~Gy} \mathrm{EQD2} 2_{(\alpha / \beta=3)}\right)$, and median $\mathrm{D}_{2 \mathrm{cc}}$ for rectum was 71.9 Gy (58.3-83.7 Gy EQD2 $(\alpha / \beta=3))$.

A typical example of a dose plane distribution is shown on Figure 3. 


\section{Discussion}

Despite medical advances, the treatment of cervix malignancies, mainly locally advanced tumors, remains one of the main clinical challenges for the radiation oncologist. External beam radiotherapy with concomitant chemotherapy and intracavitary BT represent the gold standard for these patients.

In contrast with the old point-based techniques [21], the incorporation of CT-based tridimensional imaging for BT planning has been a very important and well-known advance. It allows volume definition providing dose-volume histograms to predict potential control and toxicity.

Several studies have reported that MRI can also be an extremely useful tool for diagnosis and prediction of response in cervical malignances [22-25], and this modality has been recommended as superior to CT $[1,26]$. The ability of MRI to provide precise knowledge of disease extension has facilitated the tailoring of treatment to individual circumstances at different stages of the disease. Clearly, this characteristic of MRI-based diagnosis aids in selection of the best radiation source placement for the BT procedure and the BT-applicator that will be able to cover the entire CTV [10,27-29].
In 2004, the Image-Guided Brachytherapy Working Group of the ABS published their guidelines for image-based BT for cervical carcinoma for North America [30]. At almost the same time, several European specialist groups published reports with the GEC-ESTRO recommendations [5,6], combining the work of both societies to facilitate inter-institutional standardization [31].

The GEC-ESTRO recommendations defined different BT volumes (GTV, High risk CTV, and Intermediate risk CTV), based on the disease and risk of recurrence because of microscopic tumor at the diagnosis and at the time of the BT procedure, i.e., clinical, and as described by MRI. These volumes should receive different doses individually adapted to each application. The GEC-ESTRO recommendations have introduced the term Image-Guided Brachytherapy to indicate this stage of the treatment process. Application of these guidelines is intended to reduce toxicity and increase local control and survival; several different groups and institutions have reported on the efficacy of this approach [32-34].

Despite these efforts, inadequate dose coverage remains the main reason for local failure. It is well known that higher doses in these tumors have increased local control and survival, producing control rates near $90-95 \%$ when

Table 1. Dosimetric data of patients treated with Benidorm Template. EQD2 values includes both external beam radiotherapy and brachytherapy

\begin{tabular}{|c|c|c|c|c|c|}
\hline \multirow[t]{3}{*}{ Case } & \multirow[t]{3}{*}{ Stage/Description } & \multirow[t]{3}{*}{ EBRT (Gy) } & \multicolumn{3}{|c|}{ EQD2 (Gy) } \\
\hline & & & $\alpha / \beta=10$ & $\alpha / \beta=3$ & $\alpha / \beta=3$ \\
\hline & & & $\mathrm{D}_{90} \mathrm{CTV}$ & $\mathrm{D}_{2 \mathrm{cc}}$ bladder & $\mathrm{D}_{2 c c}$ rectum \\
\hline 1 & IIB/Medial 1/3 of vagina (> $0.5 \mathrm{~cm}$ in depth) & 50.4 & 80.4 & 77.4 & 69.9 \\
\hline 2 & IIB/Medial bilateral parametrium (> $1 \mathrm{~cm}$ in MRI) & & 81.1 & 69.8 & 66.1 \\
\hline 3 & III/Parametrium affected till pelvic wall. Hydronephrosis & 50.4 & 80.5 & 76.2 & 74.2 \\
\hline 4 & IVA/Bladder & 45 & 76.9 & 72.2 & 58.3 \\
\hline 5 & $\begin{array}{l}\text { IIB/Superior third of vagina and medial parametrium } \\
(>1 \mathrm{~cm} \text { in } \mathrm{MRI})\end{array}$ & 50.4 & 81.6 & 83.6 & 75.5 \\
\hline 6 & IIB/Medial parametrium (> $1 \mathrm{~cm}$ in MRI) & 50 & 79.6 & 74.6 & 72.7 \\
\hline 7 & IVA/Rectum. Medial $1 / 3$ of vagina & 50.4 & 78.6 & 90.8 & 83.7 \\
\hline 8 & $\begin{array}{l}\text { IIB/Superior third of vagina and medial parametrium } \\
(>1 \mathrm{~cm} \text { in } \mathrm{MRI})\end{array}$ & 45 & 73.2 & 72.3 & 67.8 \\
\hline 9 & $\begin{array}{l}\text { III/Inferior third of vagina. Right parametrium until pelvic } \\
\text { wall. Hydronephrosis. }\end{array}$ & 50 & 83.8 & 81.1 & 81.4 \\
\hline 10 & IIB/Left medial parametrium (> $1 \mathrm{~cm}$ in MRI) & 50 & 79 & 80.9 & 78.9 \\
\hline 11 & IIB/Medial 1/3 of vagina. Medial parametrium (> $1 \mathrm{~cm}$ in MRI) & 53.7 & 89.9 & 78.7 & 76.1 \\
\hline 12 & IIB/Medial $1 / 3$ of vagina (> $0.5 \mathrm{~cm}$ in depth) & 50.4 & 81.1 & 69.8 & 66.1 \\
\hline 13 & IVA/Bladder. Medial $1 / 3$ of vagina & 50.4 & 81 & 79.3 & 73.9 \\
\hline 14 & $\begin{array}{l}\text { III/Left parametrium until pelvic Wall. Right medial parame- } \\
\text { trium }\end{array}$ & 50.4 & 71.5 & 76.2 & 67.6 \\
\hline 15 & $\begin{array}{l}\text { IV/Inferior half uterus. Bilateral parametrium till pelvic wall. } \\
\text { Median } 1 / 3 \text { of vagina }\end{array}$ & 50.4 & 79.2 & 81.1 & 65.9 \\
\hline
\end{tabular}

EBRT - external beam radiation therapy, EQD2 - equivalent dose at $2 \mathrm{~Gy}, \alpha / \beta$ - alpha/beta ratio, $D_{90}$ - percent of the prescription dose covering $90 \%$ of the CTV, $D_{2 c c}$ - minimum dose to the most exposed $2 \mathrm{~cm}^{3}, M R I$ - magnetic resonance imaging 
new imaging techniques were used [2,35-37]. Brachytherapy continues to be the best treatment to increase dose in locally advanced cervical carcinoma [38-40]. MRI findings have shown that there is a group of patients, in which the areas that should be treated are excluded from the classical treatments and prescriptions; these results have revealed the limitations of exclusive use of intracavitary applicators. To our knowledge, there is no established approach to treat these extended lesions using brachytherapy alone. An alternative is to combine brachytherapy with EBRT using IMRT, and then control of image registration and dose combination are the critical points [41].

Attempts to apply this kind of combined treatment have led to the development of new applicators. An interstitial component has been added to the basic IC applicators using metallic or plastic needles compatible with MRI. The two manufactured devices that apply this solution are the classic ring (Vienna applicator) and ovoids (Utrecht applicator) $[7,8,42]$. When the extension to parametrium is moderate, these combined endocavitary and interstitial MRI applicators extend the CTV coverage using a lateral line of needles [7]. This type of coverage is inadequate in patients with more advanced disease, such as bulky parametrial extension, bulky primary disease, extensive paravaginal extension to middle or lower third of the vagina, or disease invading the bladder or rectum. In these situations more interstitial needles must be added $[4,9,10]$.

There are manufactured templates for interstitial implants, such as MUPIT with angled openings [11] or the Syed template [43]. However, the MUPIT lacks a central intracavitary component that can reach deep into the cervix and neither template is MRI compatible. Plastic needles can be used but fibrosis tissue, tumors, or bones could divert these and prevent a uniform and homogenous implant.

Reports of the use interstitial BT in locally advanced cervical carcinoma are infrequent. The few published series show the use of ultrasound [44,45], MRI [13,46], or CT $[47,48]$ for needle insertion. All of these series have used plastic or stainless steel needles for CT planning (with or without fusion with MRI imaging done without the applicator and the needles). It is well known that the unavoidable time difference between the MRI and CT scans increases the overall uncertainty and can cause discomfort for patients. In our experience, these disadvantages can be resolved through the use of the Benidorm Template, which allows all planning procedures to be based solely on MRI.

The GEC-ESTRO recommends that, when using MR imaging for image-based adaptive cervical carcinoma brachytherapy, the contouring should be done in para-axial planes [49]. In our current image protocol, we are using axial planes for contouring, however, there are some 3D protocols in progress to allow the application of these recommendations.

One problem that we found in using this applicator with both the intracavitary and the interstitial component and MRI planning was that following GEC-ESTRO volume definitions for prescription became more difficult. As mentioned earlier, different doses for the HR-CTV and the IR-CTV are defined based on different risks of recurrence. The most typical strategy in interstitial BT involves including both the GTV at diagnosis and the GTV at the time of the brachytherapy in a single CTV. In the literature, although the concept is basically the same, the specific descriptions vary greatly. Souza et al. [50] define a CTV that includes "the clinical and radiological tumor at diagnostic and at the moment of the BT". Viswanathan et al. [13] and Lee et al. [47], in reporting on a huge experience in interstitial implants and MRI imaging, define the CTV as "any regions thought by the physician to harbor microscopic disease spread based on the localization of the tumor" or "CTV-1 as the clinical evident disease by CT imaging and clinical examination, facilitated by clinical drawings, fiducial markers, and pre-brachytherapy MRI when available and CTV-2 as any adjacent region with considerable risk of microscopic disease extension". Sharma et al. [51] describe the CTV as "the line joining all the peripheral needles visible in CT between the cranial and caudal extent of the disease". Also, some groups do not describe treatment volumes at all and employ CT only to define dose points in relation to catheter reconstruction [18].

According to the literature and others institutions experience, we chose to base prescription on a single CTV without distinguishing between volumes and to consider the HR-CTV to be contained within it. The resulting dose distribution was different and tended to require a lower EQD2 than is described in EMBRACE for cervix cases. However, it must be remembered that what appears to be a relatively low median EQD2 to the CTV does not take into account the effect of delivering 6 fractions two times a day in a short overall treatment time. Our median EQD2 dose to CTV reached 79.8 Gy (71.5-89.9 Gy); this is in the range described by others (EQD2 61.6-82 Gy [13,14,18,47,50,52,54]. For OAR evaluation, we followed the GEC-ESTRO recommendations on $\mathrm{D}_{2 \mathrm{cc}}$ and the EMBRACE protocol [20] using dose constraints based on the EQD2.

From January 2006 to November 2013, 89 interstitial treatments using the MUPIT template were done at our department, 65 of these as a primary treatment for locally advanced cervical cancer. In 2006 we began to employ MRI following the recommendations of GEC-ESTRO/ $\mathrm{ABS}$ in BT procedures for early stage cervical carcinoma; in 2009 we incorporated use of the Utrecht applicator for more advanced disease. Drawing on our experience in perineal interstitial treatments, Utrecht applicators and MRI BT, we designed the Benidorm Template to combine the advantages of these approaches. To our knowledge, this is the first report of the use of a completely MRI-compatible template with a mixed intracavitary and interstitial component that allows exclusive MRI based planning.

\section{Conclusions}

The Benidorm Template has been developed and clinically implemented. The design of this template addresses the disadvantages of currently commercially available templates: the inability of the intracavitary component to reach deep into the cervix (MUPIT), and the MRI-incompatibility of these templates (MUPIT and Syed), which 
necessitates use of CT imaging for the dosimetry. The use of this new MRI-compatible template is practical and efficient, allowing improved contouring and CTV conformation and planning procedure based solely on MRI.

\section{Acknowledgments}

This study was partially supported by Lorca Marin S.A. Murcia (Spain) and it was supported in part by Spanish Government under Project No. FIS2013-42156. The authors thank Mr. David Carpenter for editorial assistance.

\section{Disclosure}

Authors declare no conflict of interest.

\section{References}

1. Viswanathan A, Dimopoulos J, Kirisits C et al. Computed tomography versus magnetic resonance imaging-based contouring in cervical cancer brachytherapy: results of a prospective trial and preliminary guidelines for standardized contours. Int J Radiat Oncol Biol Phys 2007; 68: 491-498.

2. Potter R, Dimopoulus J, Georg P et al. Clinical impact of MRI assisted dose volume adaptation and dose escalation in brachytherapy of locally advanced cervix cancer. Radiother Oncol 2007; 83: 148-155.

3. Haie-Meder C, Chargari C, Rey A et al. MRI-based low dose rate brachytherapy experience in locally advanced cervical cancer patients initially treated by concomitant chemoradiotherapy. Radiother Oncol 2010; 96: 161-165.

4. Viswanathan A, Thomadsen B. American Brachytherapy society: consensus guidelines for locally advanced carcinoma of the cervix. Part I: General principles. Brachytherapy 2012; 11: 33-46.

5. Haie-Meder C, Potter R, Van Limbergen E et al. Recommendations from Gynecological (GYN) GEC-ESTRO Working Group (I): concepts and terms in 3D image based 3D treatment planning in cervix cancer brachytherapy with emphasis on MRI assessment of GTV and CTV. Radiother Oncol 2005; 74: $235-245$.

6. Potter R, Haie-Meder C, Van Limbergen E et al. Recommendations from gynaecological (GYN) GEC ESTRO working group (II): concepts and terms in 3D image-based treatment planning in cervix cancer brachytherapy-3D dose volume parameters and aspects of 3D image-based anatomy, radiation physics, radiobiology. Radiother Oncol 2006; 78: 67-77.

7. Kirisits C, Lang S, Dimopoulus J et al. The Vienna applicator for combined intracavitary and interstitial brachytherapy of cervical cancer: design, application, treatment planning and dosimetric results. Int J Radiat Oncol Biol Phys 2006; 65: 624-630.

8. Nomden CN, de Leeuw AA, Moerland MA et al. Clinical use of the Utrecht applicator for combined intracavitary/interstitial brachytherapy treatment in locally advanced cervical cancer. Int J Radiat Oncol Biol Phys 2012; 82: 1424-1430.

9. Nag S, Erickson B, Thomadsen B et al. The American Brachytherapy Society recommendations for high dose rate brachytherapy for carcinoma of the cervix. Int J Radiat Oncol Biol Phys 2000; 48: 201-211.

10. Viswanathan A, Erickson B, Rownd J. Image guided approaches to interstitial brachytherapy. In: Gynecologic radiation therapy: novel approaches to image-guidance and management. Viswanathan A, Kiristis C, Erickson B, Potter R (eds.). Springer, Berlin-Heidelberg 2011; 247-259.

11. Martinez A, Cox RS, Edmundson GK. A multiple-site perineal applicator (MUPIT) for treatment of prostatic, anorectal, and gynecologic malignancies. Int J Radiat Oncol Biol Phys 1984; 10: 297-305.

12. Petit $S$, Wielopolski P, Rijnsdorp R et al. MR guided applicator reconstruction for brachytherapy cervical cancer using the novel titanium Rotterdam applicator. Radiother Oncol 2013; 100: 88-92.

13. Viswanathan A, Szymonifka J, Tempary-Afdhal C et al. A prospective trial of real-time magnetic resonance-guided catheter placement in interstitial gynecologic brachytherapy. Brachytherapy 2013; 12: 240-247.

14. Yoshida K, Yamazaki H, Takenaka T et al. A dose-volume analysis of magnetic resonance image-aided high dose rate image-based interstitial brachytherapy for uterine cervical cancer. Int J Radiat Oncol Biol Phys 2010; 77: 765-772.

15. Richart J, Otal A, Rodriguez S et al. A practical MRI based reconstruction method for the new endocavitary and interstitial gynecological template. PO-1021. 3 ESTRO FORUM. Barcelona. 2015. Radiother Oncol 2015; Suppl 1: 115.

16. Perez-Calatayud J, Kuipers F, Ballester F et al. Exclusive MRIbased tandem and colpostats reconstruction in gynaecological brachytherapy treatment planning. Radiother Oncol 2009; 91: 181-186.

17. Carmona V, Perez-Calatayud J, Lliso F et al. A program for the independent verification of brachytherapy planning system calculations. J Contemp Brachytherapy 2010; 2: 129-133.

18. Mahantshetty U, Shrivastava S, Kalyani N et al. Templatebased high dose rate interstitial brachytherapy in gynecologic cancers: a single institutional experience. Brachytherapy 2014; 13: 337-342.

19. Wang Y, Ye WJ, Du LH et al. Dose-volume parameters and clinical outcome of CT-guided free-hand high dose rate interstitial brachytherapy for cervical cancer. Chin I Cancer 2010; 31: 598-604.

20. EMBRACE Protocol. Version 17-01-2008. Available at: https:/ www.embracestudy.dk/UserUpload/PublicDocuments/EmbraceProtocol.pdf.

21. Zhang M, Chen T, Kim LH et al. Three-dimensional dosimetric considerations from different point A definitions in cervical cancer low-dose-rate brachytherapy. J Contemp Brachytherapy 2013; 5: 222-226.

22. Mayr NA, Yuh WT, Magnotta VA et al. Tumor perfusion studies using fast magnetic resonance imaging technique in advanced cervical cancer: a new noninvasive predictive assay. Int J Radiat Oncol Biol Phys 1996; 36: 623-633.

23. Mayr NA, Wang JZ, Lo SS et al. Translating response during therapy into ultimate treatment outcome: a personalized 4-dimensional MRI tumor volumetric regression approach in cervical cancer. Int J Radiat Oncol Biol Phys 2010; 76: 719 727.

24. Ohara K, Oki A, Tanaka YO et al. Early determination of uterine cervical squamous cell carcinoma radioresponse identifies high and low-response tumors. Int J Radiat Oncol Biol Phys 2006; 64: 1179-1182.

25. Nam H, Park W, Huh SJ et al. The prognostic significance of tumour volume regression during radiotherapy and concurrent chemoradiotherapy for cervical cancer using MRI. Gynecol Oncol 2007; 107: 320-325.

26. Durfee SM, Zou KH, Muto MG et al. The role of magnetic resonance imaging in treatment planning of cervical carcinoma. J Womens Imag 2000; 2: 63-68.

27. Tanderup K, George D, Potter R et al. Adaptive management of cervical cancer radiotherapy. Semin Radiat Oncol 2010; 94: 173-180.

28. Tanderup K, Nielsen SK, Nyvang GB et al. From point A to the sculpted pear: MR image guidance significantly improves tumour dose and sparing of organs at risk in brachytherapy for cervical cancer. Radiother Oncol 2010; 20: 121-129. 
29. Dimopoulus JC, Shard G, Berger D et al. Systematic evaluation of MRI findings in different stage of treatment of cervical cancer: potential of MRI on delineation target, pathoanatomic structures, and organ at risk. Int J Radiat Oncol Biol Phys 2006; 64: 1380-1388.

30. Nag S, Cardenes H, Chang S et al. Image-Guided Brachytherapy Working Group. Proposed guidelines for image-based intracavitary brachytherapy for cervical carcinoma: report from Image-Guided Brachytherapy Working Group. Int J Radiat Oncol Biol Phys 2004; 60: 1160-1172.

31. Nag S. Controversies and new developments in gynecologic brachytherapy: image-based intracavitary brachytherapy for cervical carcinoma. Semin Radiat Oncol 2006; 16: 164-167.

32. Potter R, Petra G, Johannes CAD et al. Clinical outcome of protocol based image (MRI) guided adaptive brachytherapy combined with 3D conformal radiotherapy with or without chemotherapy in patients with locally advanced cervical cancer. Radiother Oncol 2011; 100: 116-123.

33. Dimopoulus JC, Potter R, Lang S et al. Dose-effect relationship for local control of cervical cancer by magnetic resonance image guided brachytherapy. Radiother Oncol 2009; 93: 311-315.

34. Lindergaard JC, Fokdal LU, Nielsen SK et al. MRI-guided adaptive radiotherapy in locally advanced cervical cancer from a Nordic perspective. Acta Oncol 2013; 52: 1510-1519.

35. Dimopoulus JC, Lang S, Kristis C et al. Dose-volume histogram parameters and local tumor control in magnetic resonance image-guided cervical cancer brachytherapy. Int J Radiat Oncol Biol Phys 2009; 75: 56-63.

36. Schmid MP, Kiristis C, Nesvacil N et al. Local recurrences in cervical cancer patients in the setting of image-guided brachytherapy: a comparison of spatial dose distribution within a matched-pair analysis. Radiother Oncol 2011: 100: 468-472.

37. Nomden CN, de Leeuw AAC, Roesink JM et al. Clinical outcome and dosimetric parameters of chemo-radiation including MRI guided adaptive brachytherapy with tandem-ovoid applicators for cervical cancer patients: a single institution experience. Radiother Oncol 2013; 107: 69-74.

38. Lindegaard JC, Tanderup K. Counterpoint: time to retire the parametrial boost. Brachytherapy 2012; 11: 80-83.

39. Tanderup K, Eifel P, Yashar C et al. Curative radiation therapy for locally advanced cervical cancer: brachytherapy is NOT optional. Int J Radiat Oncol Biol Phys 2014; 88: 537-539.

40. Sharma D, Gandhi AJ, Sharma S et al. Interstitial brachytherapy vs. intensity-modulated radiation therapy for patients with cervical carcinoma not suitable for intracavitary radiation therapy. Brachytherapy 2013; 12: 311-316.

41. Mohamed S, Kallehauge J, Fokdal L et al. Parametrial boosting in locally advanced cervical cancer: Combined intracavitary/interstitial brachytherapy vs intracavitary brachytherapy plus external beam radiotherapy. Brachytherapy 2015; 14 23-28.

42. Fokdal L, Tanderup K, Hokland SB et al. Clinical feasibility of combined intracavitary/interstitial brachytherapy in locally advanced cervical cancer employing MRI with a tandem/ring applicator in situ and virtual preplanning of the interstitial component. Radiother Oncol 2013; 107: 63-68.

43. Syed A, Puthawala AA, Neblett D et al. Transperineal interstitial intracavitary "Syed-Neblett" applicator in the treatment of carcinoma of the uterine cervix. Endocuriether Hypertherm 1986; 2: 1-13.

44. Sharma DN, Rath GK, Thulkar S et al. Use of transrectal ultrasound for high dose rate interstitial brachytherapy for patients of carcinoma of uterine cervix. J Gynecol Oncol 2010; 21: $12-17$.
45. Erickson B, Foley WD, Gillin M et al. Ultrasound-guided transperineal interstitial implantation of pelvic malignances: description of the technique. Endocurie Hyperther Oncol 1995; 11: 107-113.

46. Corn BW, Lanciano RM, Rosenblum N et al. Improved treatment planning for the Syed-Neblett template using endorectal-coil magnetic resonance and intraoperative (laparotomy/ laparoscopy) guidance: a new integrated technique for hysterectomized women with vaginal tumors. Gynecol Oncol 1995; 56: 255-261.

47. Lee L, Damato A, Viswanathan A. Clinical outcomes of highdose-rate interstitial gynecologic brachytherapy using real time CT guidance. Brachytherapy 2013; 12: 303-310.

48. Erickson B, Albano K, Guillin M. CT-guided interstitial implantation of gynecologic malignancies. Int J Radiat Oncol Biol Phys 1996; 36: 699-709.

49. Dimopoulos, JC, Petrow P, Tanderup K et al. Recommendations from Gynaecological (GYN) GEC-ESTRO Working Group (IV): Basic principles and parameters for MR imaging within the frame of image based adaptive cervix cancer brachytherapy. Radiother Oncol 2012; 103: 113-122.

50. D'Souza D, Wiebe E, Patil N et al. CT-based interstitial brachytherapy in advanced gynecologic malignancies: outcomes from a single institution experience. Brachytherapy 2014; 13 : 225-232.

51. Sharma D, Rath G, Thulkar S et al. High-dose rate interstitial brachytherapy using two weekly sessions of $10 \mathrm{~Gy}$ each for patients with locally advanced cervical carcinoma. Brachytherapy 2011; 10: 242-248.

52. Kannan N, Beriwal S, Kim H et al. High dose rate interstitial computed tomography-based brachytherapy for the treatment of cervical cancer: early results. Brachytherapy 2012; 11: 408-412.

53. Fokdal L, Tanderup K, Nielsen $S$ et al. Image and laparoscopic guided interstitial brachytherapy for locally advances primary or recurrent gynecological cancer using the adaptive GEC ESTRO target concept. Radiother Oncol 2011; 100: 473-479.

54. Saitoh J, Ohno T, Sakurai H et al. High-dose rate Interstitial Brachytherapy with Computed Tomography-based Treatment Planning for patients with locally advanced uterine cervical carcinoma. J Radiat Res 2011; 52: 490-495. 